\title{
Falla ovárica prematura: Causas genéticas
}

\author{
Felipe Arbeláez C. MD.*; Mario Rebolledo A. MD.**; C.M. Restrepo MD.***
}

RESUMEN: Presentamos una paciente de 33 años de edad que consulta por amenorrea secundaria causada por una Falla Ovárica Prematura (FOP) secundaria a un Síndrome Turner en mosaico.

La FOP definida como amenorrea hipergonadotrópica en pacientes menores de 40 años tiene una incidencia del 2 al $10 \%$. Es causada por diferentes entidades entre las que encontramos desórdenes autoinmunes, defectos metabólicos y enzimáticos, enfermedades infecciosas, causas iatrogénicas, defectos en las gonadotropinas y causas genéticas. Estas últimas son responsables de FOP especialmente en pacientes menores de 30 años, en las que se justifica la solicitud de cariotipo como parte del estudio. Describimos las características de la disgenesia gonadal, del Síndrome Turner y los elementos funcionales del cromosoma $X$ cuya ausencia altera la función gonadal. También mencionamos los diferentes mosaicos descritos en relación al Síndrome Turner y sus consecuencias en el desarrollo somático y gonadal.

PALABRAS CLAVES: Falla ovárica prematura, Síndrome Turner, mosaicismo, disgenesia gonadal.

SUMMARY: We present a case of a 33 year old patient with secundary amenorrhea caused by a premature ovarian failure (FOP) secundary to a Turner's Syndrome in mosaicism.

The FOP, defined as hipergonadotropic amenorrhea in patients under 40 years of age, has an incidence between $2-10 \%$. It is caused by different entities among which we can find autoinmune disorders, metabolic and enzimatic defects, infectious diseases, iatrogenic causes, defects in the gonadotropins and genetic causes. The later are responsable for FOP specially in patients under 30 years of age, in whom it is justified to make a cariotipe as part of thier study. We describe the characteristies of gonadal dysgenesis, Turner's Syndrome and the functional elements of the $X$ chromosome whose abscense alters the gonadal function. We also mention the different mosaicisms described in relation with Turner's Syndrome and its consecuences in somatic and gonadal development.

KEY WORDS: Premature ovarian failure, Turner's Syndrome, mosaicism, gonadal dysgenesis.

\section{Introducción}

Con el presente artículo se desea revisar la falla ovárica prematura a partir de un caso clínico de la consulta de endocrinología e infertilidad del Hospital Infantil Universitario "Lorencita Villegas de Santos", haciendo énfasis en las causas genéticas.

\section{Historia clínica}

Paciente de sexo femenino, de 33 años de edad, que consulta en junio de 1993 por amenorrea secundaria de 3 años de evolución, menarquia, telarquia y pubarquia a los $12 \frac{1}{2}$ años, con ciclos regulares de $30 \times 4$ hasta los 25 años, relaciones sexuales desde los 20 años, sin planificación familiar GOPO y posteriormente presentó oligomenorreas hasta $\operatorname{los} 30$ años, a partir de los cuales se encuentra en amenorrea. En la revisión por sistemas se relatan síntomas hipoestrogénicos: como oleadas de calor, dispareunia su-

\footnotetext{
* Instructor. Departamento de Ginecología y Obstetricia. Hospital Infantil Universitario "Lorencita Villegas de Santos".

** Instructor. Departamento de Ginecología y Obstetricia. Hospital Infantil Universitario "Lorencita Villegas de Santos".

*** Unidad de Genética y Medicina Molecular. Facultad de Medicina. Colegio Mayor de Nuestra Señora del Rosario.
}

perficial y dolores osteoarticulares en las extremidades inferiores.

No ha presentado enfermedades importantes. Los padres se refieren sanos y no son consanguíneos. No hay antecedentes similares u otras heredopatías en ambas ramas parentales.

Al examen físico mostró: Peso: $61.5 \mathrm{Kg}$. Talla: 147 $\mathrm{cm}$, con índice de masa corporal de 28.3. Signos vitales normales, Talla baja, normocefalia, frente estrecha, orejas e implantación del cabello bajas, Pterigyum colli, tórax ancho con teletelia, glándulas mamarias Tanner V, valoración cardiopulmonar y abdomen normales, genitales externos normales de nulípara, Tanner $\mathrm{V}$, vello genital de distribución ginecoide. En la cervicovaginoscopia se observó vagina y cuello de aspecto sano y al tacto, vagina y cuello normales; útero en anteversoflexión, de tamaño, forma y consistencia normal, anexos sin masas y fondos de saco libres.

Los exámenes de laboratorio revelaron: química sanguínea incluyendo perfil lipídico y citología vaginal normales. Los estudios hormonales mostraron TSH en $3.5 \mathrm{mUI} / \mathrm{ml}$, PRL en $13.3 \mathrm{ng} / \mathrm{ml}$, test de supresión progestacional negativo, FSH mayor de $200 \mathrm{mUI} / \mathrm{ml}$ y LH $162 \mathrm{mUI} / \mathrm{ml}$. La densitometría ósea evidenció un contenido de Calcio del esqueleto axial normal (L2-L4) 
$1.157 \mathrm{~g} / \mathrm{cm}^{2}$ (Percentil 41). El contenido de Calcio del esqueleto apendicular también se observó normal (fémur derecho) $1.144 \mathrm{~g} / \mathrm{cm}^{2}$ (Percentil 94). El estudio de autoinmunidad informó: anticuerpos antinucleares negativos, anticuerpos antimicrosomales y antitiroglobulina también negativos. El estudio genético, realizado mediante el cultivo linfocitario de sangre periférica bajo técnicas convencionales y análisis con bandeo GTG mostró un mosaicismo cromosómico compuesto por un $84 \%$ de células normales, $46 \mathrm{XX}$ y un $16 \%$ de células con monosomía X, 45 X. Se estableció entonces el diagnóstico de Falla Ovárica Prematura secundaria a un Síndrome Turner en mosaico.

Con este diagnóstico se inició terapia de suplencia hormonal con estrógenos naturales conjugados, progestágenos y calcio y se indicó ejercicio.

\section{Falla ovárica prematura}

Definición. La falla ovárica prematura (FOP) es un síndrome heterogéneo caracterizado por amenorrea primaria o secundaria, hipergonadotrofismo en mujeres menores de 40 años, asociados a hipoestrogenismo (1-3): Algunos autores, actualmente prefieren denominarla amenorrea hipergonadotrópica por la forma de presentación, evolución clínica y pronóstico (3-5).

Es una condición en la cual los ovarios han logrado su diferenciación y función adecuada, pero ésta se agota antes del momento esperado de la menopausia, lo cual, genera un compromiso emocional y reproductivo de importancia significativa (1).

\section{Incidencia}

La incidencia de la FOP no ha sido bien establecida. Se estima que representa entre el 2 al $10 \%$ de las causas de amenorrea primaria o secundaria, y afecta del 1 al $3 \%$ de la población general $(1,16)$. En la Unidad de Investigación Clínica en Reproducción Humana del "Hospital Infantil Lorencita Villegas de Santos", la FOP representa cerca del $2 \%$ de la consulta de endocrinología e infertilidad (Barón G. Arbeláez F.).

\section{Diagnóstico}

El cuadro clínico se caracteriza por amenorrea primaria en el 15\% y secundaria en el $85 \%$ de los casos; además, se observan síntomas de hipoestrogenismo como oleadas de calor, atrofia genital y compromiso de la esfera psicoafectiva. Existe una falla en el proceso de desarrollo sexual secundario hasta en $20 \%$ de los casos y en el $75 \%$ hay infertilidad $(1-2,4)$.

En los esquemas usuales de evaluación de la paciente con amenorrea se deben tener en cuenta que el test de supresión progestacional puede dar falso positivo hasta en un $50 \%$ de las pacientes (3-5).

En la evaluación paraclínica, se observan niveles de FSH mayores de $40 \mathrm{mIU} / \mathrm{ml}$, una relación $\mathrm{LH} / \mathrm{FSH}$ menor de 0.7 (debido a la elevación hasta en 10 veces en los niveles de FSH y sólo de 3 veces en los niveles de LH). El nivel sérico de estradiol es similar al de la fase folicular temprana, es decir menor de $45 \mathrm{pg} / \mathrm{ml}(1-5,10)$.

\section{Etiología}

Al mencionar las causas de la pérdida acelerada de folículos que ocurre en las pacientes con FOP, es necesario entender el proceso de gonadogénesis del ovario. Para un adecuado desarrollo ovárico se requiere la presencia de 2 cromosomas sexuales $\mathrm{X}$ normales y la ausencia del cromosoma Y. En la gónada destinada a ser ovario, el proceso de diferenciación se inicia hacia las 11 a 12 semanas de vida intrauterina, cuando las células germinales que han migrado desde el endodermo del saco vitelino a la cresta gonadal, se diferencian de oogonia en oocito empezando su división meiótica. Hacia la semana 20, se alcanza el máximo número de oocitos (aproximadamente 7 millones) y a partir de este momento empieza un proceso de atresia, de tal forma que al nacimiento existen 2 millones de oocitos, y en la pubertad persisten cerca de 400.000 que suplen a la mujer durante la vida reproductiva hasta su agotamiento en la menopausia (2, 6-9).

Desde el punto de vista fisiopatológico se pueden considerar tres mecanismos para la pérdida de oocitos: el primero, es un proceso de la atresia fisiológica descrita; el segundo, es una disminución de la dotación de células germinales al nacimiento; y el tercero, es la destrucción de los oocitos en cualquier momento de la vida $(2,10)$.

Es importante mencionar algunos aspectos relevantes de las diferentes causas descritas de FOP con énfasis especial en las genéticas, motivo fundamental de esta publicación:

Causas inmunológicas. La FOP se asocia entre un 13 a un $65 \%$ de los caos con algunos desórdenes autoinmunes, entre otros, patologías de tiroides (el desorden glandular más frecuentemente), la enfermedad de Addison, el síndrome Sicca, la artritis reumatoidea, el hipoparatiroidismo, la miastenia gravis, la diabetes mellitus, la púrpura trombocitopénica inmunológica y otras (1-5). Se han descrito diferentes tipos de anticuerpos contra el tejido ovárico (tanto para el oocito, como para las células de la teca y la granulosa), las gonadotropinas y su receptor (1, 3, 14-15). También se han encontrado defectos en la función de las células involucradas en la respuesta inmune (1). Además, se han reportado una asociación significativa con el HLA-DR3, que implica una susceptibilidad genética a la autoinmunidad $(3,13)$.

Causas iatrogénicas. Son tres los agentes involucrados en el proceso de suspensión de la función gonadal: cirugía, radioterapia y quimioterapia.

De los quimioterápicos involucrados son los alquilantes, especialmente la ciclofosfamida, la más comprometida y afecta más a las pacientes de mayor edad. La radioterapia a dosis superiores a 800 rads produce una falla ovárica irreversible casi el $100 \%$ de los casos.

Cuando algunas pacientes recuperan la función endocrina y reproductiva después de quimioterapia o radioterapia no hay ningún efecto sobre sus productos en lo que respecta a anomalías congénitas, excepto en casos de exposición a la actinomicina-D, que se ha asociado con un riesgo elevado de enfermedad cardíaca congénita. En cuanto a la cirugía, además de la ooforectomía per se, el 
compromiso vascular o la lesión del tejido ovárico pueden llevar a FOP $(1-3,5,9-12)$.

Causas metabólicas y enzimáticas. La galactosemia, un desorden autosómico recesivo, caracterizado por deficiencia de la enzima galactosa-1-fosfato uridiltransferasa, produce niveles anormalmente elevados de galactosa, galactitol y galactosa-1-fosfato a nivel intracelular, al parecer elementos tóxicos para el ovario fetal, efecto que no se puede evitar aún con dieta libre de galactosa desde la infancia. El diagnóstico se hace mediante la demostración de una elevación sérica de galactosa y la determinación enzimática en los leucocitos. La restricción de la ingesta de galactosa desde la infancia no evita la destrucción de los oocitos pero es probable que esa misma restricción a la madre durante el embarazo si evite el daño del ovario fetal (1-3, 5, 11-12).

Las pacientes con deficiencia de $17 \mu$ hidroxilasa (requerida para la síntesis de estrógenos y cortisol), que sobreviven hasta la adolescencia, se presentan con hipertensión, alcalosis hipocalémica y ausencia de caracteres sexuales secundarios con FOP (1-3, 5, 11-12).

Desórdenes infecciosos: Son una causa infrecuente y difícil de confirmar. El virus de la Parotiditis (virus ARN de la familia paramyxovirus), puede producir citólisis y reacción inflamatoria en el tejido ovárico, especialmente en la vida fetal y puberal (1-3, 5, 11-12, 20).

Las pacientes con tuberculosis pélvica pueden tener destrucción ovárica en el 3\% de los casos (1, 21); igualmente, se han descrito casos aislados de FOP en pacientes con malaria y shiguellosis (12).

Defectos en la estructura y/o acción de las gonadotropinas. En algunos casos de FOP se han encontrado anormalidades èn la estructura, secreción o metabolismo de las gonadotropinas, con una actividad biológica reducida o ausente que llevará a atresia acelerada de los folículos. También se han descrito alteraciones en la acción de la FSH por cambios en la estructura de su receptor y la presencia de inhibidores competitivos $(1,3$, 5, 11-12).

Causas genéticas. Son una de las más frecuentes entre los desórdenes conocidos de FOP (1), especialmente en las mujeres menores de 30 años. Está justificada la solicitud del cariotipo en todas estas pacientes, con el objeto de aclarar la etiología y descartar la presencia de cromosomas Y (1-6, 9-12, 16-18); además, en este último caso, por el riesgo de malignización gonadal hacia gonadoblastoma o disgerminoma, observado hasta en $25 \%$ de los casos y con frecuencia antes de los 25 años $(6,28-32)$.

La FOP causada por alteraciones cromosómicas puede manifestarse por amenorrea primaria (55\% de los casos) y secundaria en el $13 \%$ (4). Ya hemos hablado de la importancia de la presencia de 2 cromosomas $X$ intactos para el mantenimiento normal de los oocitos, de tal forma que las alteraciones estructurales o numéricas de este cromosoma van a manifestarse por anormalidades del desarrollo gonadal. Los cariotipos más frecuentemente observados son:
- 45, X.

- Mosaicos 45,X/46,XX (alrededor de 27\%).

- $47, \mathrm{XXX}(25 \%)$.

- Alteraciones estructurales del tipo de deleciones, translocaciones, isocromosomas $(23 \%)$.

La disgenesia gonadal se ha definido tradicionalmente con base en el hallazgo fenotípico de gónadas atrésicas o en banda, evidenciándose alteraciones en los cromosomas en la mayoría de los casos. Sin embargo, en algunos individuos no se detectan aberraciones cromosómicas, aún con técnicas de Alta Resolución, probablemente por la presencia de deleciones submicroscópicas o alteraciones por inactivación de un cromosoma X (22-23).

En 1938, Henry H. Turner describió un grupo de 7 pacientes con infantilismo sexual, cuello alado y cubitis valgus en la revista Endocrinology (24), pero sólo hasta 1959 Ford encontró que el constituyente cromosómico de la paciente típica con el Síndrome Turner era 45,X (26).

El brazo corto ( $p$ ) del cromosoma $X$ y el brazo corto del cromosoma Y tienen genes que poseen la información necesaria para un adecuado crecimiento y normal desarrollo esquelético y cardiovascular; su ausencia ocasiona los estigmas somáticos típicos del Síndrome Turner. De manera similar, tanto el brazo largo como en el corto del cromosoma $X$, se encuentran los genes que modulan el desarrollo y la función gonadal $(6,9,23,25,27)$.

Los estudios de Race y Sanger demostraron que el $75 \%$ de los cromosomas $X$ en las pacientes $45, X$ son de origen materno, lo cual significa que en la mayoría de los casos el otro cromosoma $X$ se perdió por no disyunción en la meiosis paterna, efecto independiente de la edad del progenitor (23).

Los fetos con gónadas 45,X tienen un complemento normal de oocitos en las semanas 20 a 24 , pero éstos entran en un proceso de atresia acelerada de forma tal que el nacimiento no se encuentra dotación folicular $(5-6,17)$.

No se conoce la razón por la cual los fetos $45, X$ tienen predisposición a abortarse, siendo la causa del $10 \%$ de todas las pérdidas del primer trimestre y aproximadamente el $1.5 \%$ de todas las concepciones humanas, de tal suerte que por esta selección prenatal natural, al nacimiento su incidencia es de 1 en 10.000 en la forma clásica. Además, una de cada 2.500 a 3.500 recién nacidas vivas tienen una aberración cromosómica que puede llevar a disgenesia gonadal $(6,23,25)$.

Las características clínicas más frecuentes observadas en las pacientes con el cariotipo $45, \mathrm{X}$ se describen en la Tabla 1 .

De las pacientes con síndrome de Turner, el 40 a $60 \%$ tienen monosomía $X$. Las restantes tienen una variedad de cariotipos que incluyen alteraciones estructurales de los cromosomas $\mathrm{X}$ y $\mathrm{Y}$ e incluso autosomas (deleciones, anillos, translocaciones e isocromosomas), diferentes formas de mosaicos que involucran una o más líneas celulares con anormalidades en el número. La más frecuente de las anomalías estructurales es el isocromosoma $\mathrm{X}$ $(46, \mathrm{Xi}(\mathrm{Xq}))(6,23,25)$.

Los mosaicos se encuentran en el $40 \%$ de las pacientes con síndrome de Turner aproximadamente, siendo los más comunes el 45,X/46,XX ó 45,X/47,XXX (15\%), 45,X/ $46, X, \mathrm{i}(\mathrm{Xq})(10 \%)$ y otros (ver tabla 2$)(6,23,25)$. Un 
estudio nacional mostró en 38 pacientes con Turner los cariotipos descritos en la Tabla 3 (27).

\section{Tabla 1}

ALTERACIONES SOMATICAS ASOCIADAS FRECUENTEMENTE CON EL CARIOTIPO 45,X

\begin{tabular}{|l|}
\hline Cráneofaciales \\
Epicanto (25\%) \\
Paladar ojival (36\%) \\
Dientes anormales \\
Alteraciones visuales (estrabismo) (22\%) \\
Defectos auditivos (defectos del oído interno) \\
\hline Cuello \\
Pterygium colli (46\%) \\
Cuello corto, ancho, con implantación baja del cabello \\
(74\%) \\
\hline Tórax \\
Tórax ancho con areolas aparentemente muy separadas \\
(53\%) \\
Cardiovascular (10-16\%) \\
\hline Coartación de la aorta \\
Defectos del tabique interventricular \\
\hline Renales (38\%) \\
Riñón en herradura \\
Duplicación ureteral \\
Aplasia o hipoplasia renal unilateral \\
\hline Gastrointestinales \\
Telangiectasias \\
\hline Piel y linfáticos \\
Nevus pigmentados (63\%) \\
Linfedema de manos y pies (38\%) \\
\hline Uñas \\
Hipoplasia o malformación (66\%) \\
\hline Esqueleto \\
Cubitus valgus (54\%) \\
Metacarpianos o metatarsianos cortos (IV) (48\%) \\
Deformidad del cóndilo tibial medial \\
Osteoporosis \\
\hline
\end{tabular}

Se define mosaico a la presencia de por lo menos 2 líneas celulares con cariotipos diferentes derivados de un único cigoto, el estado de desarrollo en el cual ocurre una segregación anormal se cree que determina la proporción y la distribución de las diferentes líneas celulares $(6,23)$. Se cree que el mosaico es una consecuencia directa de la inestabilidad mitótica de los cromosomas anómalos. La relación de células germinales primordiales con cariotipos 45,X y 46,XX ó 46,XY determina el estado de la gónada, mientras que la misma proporción en los tejidos periféricos determina el fenotipo general (6).

Las manifestaciones clínicas en el mosaico tienden a ser más leves, encontrándose con mayor frecuencia pacientes que menstruan - $20 \%$ en comparación al $10 \%$ en las monosomias-, mayor éxito reproductivo, mayor estatura y menor frecuencia de algunas anormalidades fenotípicas $(23,28,34-35)$. Los pacientes con mosaico del Síndrome
Tabla 2

CARIOTIPOS MOSAICO ASOCIADOS CON SINDROME TURNER

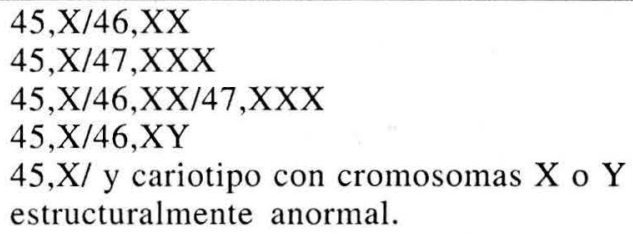

Tabla 3

CARIOTIPOS SINDROME TURNER

\begin{tabular}{|c|c|}
\hline Cariotipo & Porcentaje \\
\hline $\begin{array}{l}\text { Monosomía X (clásica) } \\
45, X\end{array}$ & 52.6 \\
\hline $\begin{array}{l}\text { Mosaicos } \\
45, X / 46, X X\end{array}$ & 2.6 \\
\hline $\begin{array}{l}\text { Isocromosoma Xq } \\
45, X / 46, X, i(X q) \\
45, X / 46, X, i(X q) / 47, X, i(X q), i(X q) \\
46, X i(X q) \\
45, X / 46, X, i d i c(X q)\end{array}$ & 26.3 \\
\hline $\begin{array}{l}\text { Deleción } \mathrm{Xp} \\
46, \mathrm{X} \text {, del }(\mathrm{Xp}) \text { ó } 46, \mathrm{X} \text {, del }(\mathrm{Xp})\end{array}$ & 2.6 \\
\hline $\begin{array}{l}\text { Anillos } X \\
45, X / 46, X, r(X) \\
45, X / 46, X, r(X) / 46, X, f(X)\end{array}$ & 7.9 \\
\hline $\begin{array}{l}\text { Cromosoma Y } \\
45, X / 46, X, i d i c(Y q) \\
45, X / 46, X Y^{n f}\end{array}$ & 5.3 \\
\hline $\begin{array}{l}\text { Fragmentos } X \\
45, X / 46, X, f(X)\end{array}$ & 2.6 \\
\hline
\end{tabular}

Cariotipos encontrados en 38 pacientes con Síndrome Turner. Estudio nacional. Giraldo A, Silva E, Bueno ML et al. Biomédica 1983; 3: 107-117.

Turner $45, X / 46, X X$, se presentan con pocos estigmas, una talla mayor en comparación a las pacientes con monosomía y, además, un alto porcentaje tienen un desarrollo sexual secundario, una función menstrual normal y las gónadas pueden ser en banda, hipoplásicas o normales $(6,28,31)$. Se ha reportado infertilidad, pérdidá fetal repetida y éxito reproductivo en pacientes con monosomías y mosaicos $(6,34-36)$. La preservación de la función ovárica en estas pacientes se debe a que poseen una línea celular con el otro cromosoma X normal o con un rearreglo estructural que no compromete la "región crítica" en el cromosoma $(35,37)$. Cuando las alteraciones cromosómicas ocurren en la descendencia de estas pacientes se observa similitud en sus cariotipos.

Casos idiopáticos. Es un diagnóstico desafortunadamente frecuente en la práctica clínica. 


\section{BIBLIOGRAFIA}

1. Cohen I., Speroff L. Premature ovarian failure: Update. Obstet. Gynecol. Surv. 1991; 46: 156-162.

2. Asare T.,EdwardEW. Premature ovarian failure. Post. Obstet. Gynecol. 1993; 13(19): 1-4

3. Rebar WR., Cedars Ml. Hipergonadotropic forms of amenirrhea in young women. En Moghissi KS. Endocrenology and Metabolism Clinics of North America: Reproductive Endocrinology. 1992; 1: 173-191.

4. Rebar WR., Connolly HV. Clinical features of young women with hypergonadotropic amenorrhea. Fertil Steril 1990; 53: 804-810.

5. Rebar WR. Premature ovarian failure. En: Lobo RA, ed. Treatment of the Postmenopasual Women: Basic and clinical Aspect. First edition. New York: Raven Press, Ltd., 1994; 25-34.

6. Conte FA., Grumbach MM. Pathogenesis, classification, diagnosis, and treatment of anomalies of sex. En: De Groot, ed. Endocrinology. Volumen 3. Second edition. W. B. Saunders; 1989; 1810-1841.

7. Moore KL. Embriología Clínica. Primera Edición. Interamericana; $1985 ; 197-235$.

8. Langman J. Sistema urogenital. En: Embriología Médica. Cuarta Edición. Panamericana; 1981; 235-265.

9. Soules MR., Pagon RA., Burns MW et al. Normal and abnormal sexual development. En Carr BR., Blackwell RE, eds. Textbook of Reproductive Medicine. Norwalk, Connecticut: Appleton \& Lange; 1993; 67-88.

10. Styne DM., Grumbach MM. Disorders of puberty in the male and female. En: Yen SSC., Jaffe RB, eds. Reproductive Endocrinology. Third edition. Philadelphia: W.B. Saunders Company 1991; 511-554.

11. Mesa N. Falla ovárica prematura. Unidad de investigación clínica en reproducción humana. Hospital Infantil 1994 (trabajo sin publicar).

12. Rebolledo M. Falla ovárica prematura. Unidad de investigación clínica en reproducción humana. Hospital Infantil 1993 (trabajo sin publicar).

13. Eisenbarth GS. Autoinmune Endocrine Disorders. En: De Groot LJ, ed. Endocrinology. Volumen 3. Second Edition: W.B. Saunders Company, 1989; 2632-2648.

14. Moncayo H., Moncayo R., Benz R et al. Ovarian failure and autoimmunity: Detection of autoantibodies direct egainst both the unoccupied luteinizing hormone/human chorionic gonadotropin receptor and the hormone-receptor complex of bovine corpus luteum. J. Clin Invest 1989; 84: 1857-1861.

15. Kreiner D., Droesch K., Navot D et al. Spontaneous and pharmacologically induced remissions in patients with premature ovarian failure. Obstet. Gynecol. 1988; 72: 926-930.

16. Coulam CB., Adamson SC., Annegers JF. Incidence of ovarian failure. Obstet. Gynecol. 1986; 67: 604-610.

17. Speroff L., Glass RH., Kase NG. Normal and abnormal sexual development. En: Clinical Gynecologic Endocrinology and Infertility. Fifth Edition. Baltimore: Williams \& Wilkins; 1994; 346-350.

18. Vaurell., Calaf J., Balaseli. Fertilidad y esterilidad humanas. Primera edición. Barcelona: Salvat Editores; 1992; 59-77.
19. Hayden FG., Halperin SA., Paramixovirus. En: Cline MJ., Daly WJ., Easton JD et al. eds. Internal Medicine Jay H. Stein. First Edition. Boston: Little, Brown and Company; 1983; 1321-1323.

20. Ray CG., Mumps En: Brauwald E., Isselbacher KJ., Petersdorf RG et al. eds. Harrison's Principlesof Internal Medicine. Eleventh Edition. New York: McGraw-Hill Book Company 1987; 709-712.

21. Jones HW., Jones GS. Tuberculosis genital. En: Tratado de Ginecología de Novak. Décima edición. México DF., Interamericana; $1988 ; 488-489$.

22. Stradtman EW. Genetics in reproduction. En: Carr BR., Blackwell RE eds. Textbook in Reproductive Medicine. Norwalk, Connecticut. Appleton \& Lange; 1993; 89-109.

23. De La Chapelle A. Sex chromosome abnormalities. En: Emery AEH., Rimoin DL, eds. Principles and Practice of Medical Genetics. Volumen 1. London: Churchill Levingstone; 1983; 193-215.

24. Turner HH. A syndrome of infantilism, congenital webbed neck, and cubitus valgus. Endocrinology 1938; 23: 566-574.

25. Jones KL. Síndrome XO (Síndrome de Turner). En: Atlas de Malformaciones Congénitas. Cuarta Edición: Interamericana; 1990; 79-81.

26. Ford CE., Jones KW., Polari LE et al. A sex chromosome anomaly in a clase of gonadal dysgenesis. Lancet 1959; 1: 711-715.

27. Giraldo A., Silva E., Bueno ML et al. Estudio citogenético en 38 pacientes con síndrome de Turner. Biomédica 1983; 3: 107-117.

28. Shawker TH., Garra BS., Loriaux Let al. Ultrasonography of Turner's Syndrome. J. Ultrasound Med. 1986; 5: 125-129.

29. Manuel M., Katayama KP., Jones HW. The age of occurrence of gonadal tumors in intersex patients with a Y chromosome. Am. J. Obstet. Gynecol. 1976; 124: 293-299.

30. Danahoe PK., Crawford JD., Hendren WH. Mixed gonadal dysgenesis, pathogenesis, and management. J. Ped. Surg. 1979; 14: 267-300.

31. Schellhas HF. Malignant potential of the dysgenetic gonad. Obstet. Gynecol. 1974; 44: 298-308.

32. Shah KD., Kaffe S., Gilbert F et al. Unilateral microscopic gonadoblastoma in a prepubetal Turner mosaic with $\mathrm{Y}$ chromosome material identified by restriction fragment analyssis. Am. J. Clin. Pathol. 1988; 90: 622-627.

33. Portuondo JA., Barral A., Neyro JL et al. Gonadal dysgenesis and somatic stigma in patients with $45, \mathrm{X} / 46, \mathrm{Xr}(\mathrm{X})$ ring chromosome. Int. J. Gynaecol. Obstet. 1984; 22: 311-313.

34. Baudier SMM., Chihal J., Dickey RP. Pregnancy and reproductive function in a patient with nonmosaic Turner syndrome. Obstet. Gynecol. 1985; 65: 60S-64S.

35. Ayuso MC., Bello MJ., Benitez J et al. Two fertile woman in a family. Clin Genetics 1984; 26: 591-596.

36. Hsu LYF., Garcia P., Grossman D et al. Fetal wastage and maternal mosaicism 1972; 40: 98-103.

37. Sarto GE., Therman E., Patau K. X inactivation in man: A woman with $t(X q-: 12 p)$. Am. J. Hum. Genet. 1973; 25: 262-267. 